Research Article

\title{
Diffusion-Weighted Imaging-Magnetic Resonance Imaging Information under Class-Structured Deep Convolutional Neural Network Algorithm in the Prognostic Chemotherapy of Osteosarcoma
}

\author{
Yong Hu $\mathbb{D}$, Jie Tang $\mathbb{D}$, Shenghao Zhao $\mathbb{D}$, and Ye Li $\mathbb{1}$ \\ Department of Orthopaedics, Wuhan Fourth Hospital, Wuhan 430000, Hubei, China \\ Correspondence should be addressed to Ye Li; rm001225@whu.edu.cn
}

Received 5 June 2021; Revised 4 August 2021; Accepted 6 August 2021; Published 29 August 2021

Academic Editor: Gustavo Ramirez

Copyright (c) 2021 Yong $\mathrm{Hu}$ et al. This is an open access article distributed under the Creative Commons Attribution License, which permits unrestricted use, distribution, and reproduction in any medium, provided the original work is properly cited.

\begin{abstract}
In order to improve the efficiency of early imaging diagnosis of patients with osteosarcoma and the effect of neoadjuvant chemotherapy based on the results of imaging examinations, 48 patients with suspected osteosarcoma were selected as the research objects and their diffusion-weighted imaging (DWI)-magnetic resonance imaging (MRI) images were regularized in this study. Then, a DWI-MRI image discrimination model was established based on the class-structured deep convolutional neural network (CSDCNN) algorithm. The peak signal-to-noise ratio (PSNR), mean square error (MSE), and edge preserve index (EPI) were applied to evaluate the image quality after processing by the CSDCNN algorithm; the accuracy, recall rate, precise rate, and $F_{1}$ score were employed to evaluate the diagnostic efficiency of CSDCNN algorithm; the apparent diffusion coefficient (ADC) was adopted to evaluate the therapeutic effect of neoadjuvant chemotherapy based on the CSDCNN algorithm, and SegNet, LeNet, and AlexNet algorithms were introduced for comparison. The results showed that the PSNR, MSE, and EPI values of DWI-MRI images of patients with osteosarcoma were 29.1941, 0.0016 , and 0.9688 , respectively, after using the CSDCNN algorithm to process the DWI-MRI images. The three indicators were significantly better than other algorithms, and the difference was statistically significant $(P<0.05)$. According to the results of imaging diagnosis of patients with osteosarcoma, there was no significant difference between the assisted diagnosis effect of the CSDCNN algorithm and the pathological examination results $(P>0.05)$. The results of adjuvant chemotherapy based on the CSDCNN algorithm found that the $\mathrm{ADC}_{\text {mean }}$ value of the patients after chemotherapy was $1.66 \pm 0.17$ and the $\mathrm{ADC}_{\min }$ value was $1.33 \pm 0.15$; the two indicators were significantly higher than other algorithms, and the difference was statistically significant $(P<0.05)$. In conclusion, the CSDCNN algorithm had a good effect on DWI-MRI image processing of patients with osteosarcoma, which could improve the diagnostic accuracy of patients with osteosarcoma. Moreover, the diagnosis results based on this algorithm could achieve better neoadjuvant chemotherapy effects and assist clinicians in imaging diagnosis and clinical treatment of patients with osteosarcoma.
\end{abstract}

\section{Introduction}

Osteosarcoma is a common primary malignant tumor originating from interlobular tissues and mainly occurring in adolescents and children. The patients are mostly male and tend to occur in the long bones of the extremities, with high metastasis, strong malignancy, and high disability and mortality rate $[1,2]$. Osteosarcoma mostly originates from the medullary cavity and invades the surrounding bone, and then, the tissue around the vasculature infiltrates and spreads beyond the bone to reach the outside of the bone. The periosteum is continually stimulated to form a new bone, which then breaks through and invades the surrounding soft tissue [3]. The etiology of osteosarcoma is still unclear. Some studies have reported that the incidence of osteosarcoma may be related to radiation genetic viruses and malignant transformation of benign bone disease, but no clinical studies have confirmed that human osteosarcoma is 
induced by viral infection [4]. Osteosarcoma accounts for about $15 \%$ of primary bone tumors, and its site varies slightly with the patient's age. Generally, patients under 30 years old are more likely to develop osteosarcoma in long tube bones, while patients over 50 years old are more likely to develop osteosarcoma in flat bones [5]. In addition, it is difficult to make early diagnosis with rapid growth, and patients are often accompanied with the typical clinical symptoms such as pain, soft tissue mass, and motor dysfunction. The first diagnosis is usually characterized by persistent limb pain. When the tumor breaks through the bone cortex and stimulates the periosteum, it presents persistent severe pain, which involves the joints and causes joint pain and dysfunction. In addition, some patients may have pathological fractures $[6,7]$.

Osteosarcoma causes great psychological and physiological pressure to patients. With the continuous development of medical technology, the clinical treatment of osteosarcoma has changed from traditional surgical treatment to preoperative neoadjuvant chemotherapy combined with extensive resection and postoperative chemotherapy, which has greatly improved the limb salvage rate and five-year survival rate of patients [8]. Preoperative neoadjuvant chemotherapy can effectively kill the micro-metastases, reduce the invasion of the local primary lesion on the soft tissue, and make the tumor boundary clear, thus increasing the success rate of limb salvage surgery [9]. Complete surgical resection is the key to cure osteosarcoma. At present, clinical surgical treatment of osteosarcoma focuses on obtaining an ideal resection boundary, which aims to remove the tumor to the maximum extent while protecting normal tissues, and bone and soft tissue reconstruction can be performed for bone or joint defects caused by surgical resection [10]. In addition, radiotherapy, gene therapy, and thermal and cold ablation therapy are not used as routine treatment methods but only as adjuvant treatment in the comprehensive treatment mode of "preoperative neoadjuvant chemotherapy + extensive resection + postoperative chemotherapy" [11].

The preoperative neoadjuvant chemotherapy in patients with osteosarcoma will lead to necrosis of tumor cells in the primary lesions, so the tumor necrosis rate (TNR) is generally considered the pathological gold standard to evaluate the therapeutic effect of neoadjuvant chemotherapy in osteosarcoma. However, TNR detection is complicated and sampling requirements are strict. Moreover, the TNR is only applicable to tumors acquired after radical surgery, so there is a certain lag in evaluating the therapeutic effect of osteosarcoma with TNR $[12,13]$. Therefore, imaging examination is still the main method in clinical diagnosis and treatment of osteosarcoma. The most commonly used examinations include digital radiography (DR), computed tomography (CT), and magnetic resonance imaging (MRI). Imaging of osteosarcoma presents different pathological features according to different examination methods, but such examination methods have some problems due to technical constraints, such as CT examination cannot accurately measure the length of intramedullary lesion of osteosarcoma and MRI examination is difficult to accurately reflect the metabolic characteristics of tumor tissue [14].
With the continuous innovation and development of technology, diffusion-weighted imaging (DWI) is used as a MR functional imaging method that can reflect the movement of water molecules in living tissues [15], which has been increasingly widely applied in the musculoskeletal system. DWI-MRI can display the relationship between the morphology of musculoskeletal system lesions and surrounding tissues and has a certain value in the identification of osteosarcoma. However, DWI-MRI is prone to produce magnetic sensitive artifacts and blur effect due to the limitation of sampling time in the direction of phase coding, resulting in poor image quality, low signal-to-noise ratio (SNR), and unstable value of apparent diffusion coefficient (ADC) [16]. In order to achieve the diagnostic accuracy of early imaging of patients with osteosarcoma, and to improve the effect of neoadjuvant chemotherapy for patients with osteosarcoma, this study improved the CNN algorithm and constructed a diagnostic model based on the CSDCNN algorithm. This study was designed to assist clinicians to enhance the effect of chemotherapy in patients with osteosarcoma.

\section{Materials and Methods}

2.1. Research Objects. In this study, 48 patients with suspected primary osteosarcoma admitted to hospital from January 2019 to January 2021 were selected as the research objects, including 30 males and 18 females, aged 8-32 years, with an average age of 17 years. All patients had no contraindications on MRI scanning and were confirmed by case puncture examination after the examination. This study was approved by the Ethics Committee of Hospital, and all the patients and their family members were informed about the content of this study and signed the informed consent forms.

The criteria for inclusion were defined to include patients who were newly diagnosed and had no history of surgery or chemotherapy before participating in this study; had no contraindications to MRI examination and all underwent MRI examination preoperatively; and did not suffer from tumor, infection, blood system, and other systemic diseases.

The criteria for exclusion were defined to include patients who lacked basic patient information, had a history of surgery or chemotherapy before participating in this study, and were difficult to cooperate with the treatment or did not follow the standard chemotherapy surgery treatment.

2.2. Imaging Examination Methods. All patients received the first MRI examination before puncture or surgical biopsy, and there was the second MRI examination 7 days before surgery after completing 4 cycles of neoadjuvant chemotherapy. The SIEMENS Avanto 3.0T scanner was used for MRI examination. Patients were placed in supine position, and special cores were selected according to the lesion sites of the patients. Then, they received the plain scanning of the transverse axis of T1-weighted imaging (T1WI) and T2WI and the sagittal fat-inhibition T2WI. Afterward, they underwent the enhanced scanning of the T1WI fat-inhibition transverse axis. The scanning range included tumor 
segments of the patient's affected limbs and knee joints. T1WI SE sequence: time to repetition (TR): $500-660 \mathrm{~ms}$ and time to echo (TE): $10-15 \mathrm{~mm}$; T2WI FSE sequence: TR: 2,800-4,000 ms and TE: $80-120 \mathrm{~ms}$; and matrix: $256 \times 196$, layer thickness: $5 \mathrm{~mm}$, and layer spacing: $1 \mathrm{~mm}$. DWI was applied to scan the sagittal position, with single excitation plane echo sequence. Besides, TR was $3,800 \mathrm{~ms}$, TE was $88 \mathrm{~ms}$, number of excitations was 2 , matrix was $256 \times 196$, layer thickness was $5 \mathrm{~mm}$, layer spacing was $1 \mathrm{~mm}$, and diffusion sensitivity coefficient $b$ value was taken between 0 and $800 \mathrm{~s} / \mathrm{mm}^{2}$.

\subsection{Regularization of DWI-MRI Images in Patients with} Osteosarcoma. Due to the limitation of upsampling time, DWI-MRI images of patients with osteosarcoma have a low SNR and poor image resolution is caused by the influence of physiological noise [17], which affects the subsequent analysis of clinicians. In order to denoise the images and effectively improve the SNR, the DWI-MRI images of patients with osteosarcoma were first denoising in this study. According to the imaging principle of DWI-MRI, the noise in DWI could be divided into three noise models.

Suppose there was a DWI image $S$, which could be expressed as $P=\left\{P_{1}, P_{2}, P_{3}, \ldots, P_{n}\right\}$, its Gaussian noise model can be expressed as follows:

$$
\widehat{P}_{i}=P_{i}+N_{i}=P_{0} e^{-b g_{i}^{T} D g_{i}}+N_{i} .
$$

In equation (1), $N_{i}$ represents the Gaussian white noise, whose mean value is 0 and variance is $\sigma^{2}$.

The logarithmic Gaussian noise model can be expressed as

$$
\log \widehat{P}_{i}=\log P_{0}-b g_{i}^{T} D g_{i}+N_{i}^{l} .
$$

In the above equation, $N_{i}^{l}$ stands for the Gaussian white noise, whose mean value is 0 and variance is $\sigma^{2}$.

What's more, the Rician noise model can be expressed as follows:

$$
\widehat{P}_{i}=\sqrt{\left(P_{i}+N_{1 i}\right)^{2}+N_{2 i}} .
$$

In equation (3), $N_{1 i}$ and $N_{2 i}$ represent the independent white Gaussian noises and the probability density function of $\widehat{P}_{i}$ can be calculated as follows:

$$
f_{\widehat{p}}\left(\widehat{P}_{i} \mid P_{i} ; \sigma\right)=\frac{\widehat{P}_{i}}{\sigma^{2}} \exp \left(-\frac{\widehat{P}_{i}^{2}+P_{i}^{2}}{2 \sigma^{2}}\right) I_{0}\left(\frac{\widehat{P}_{i} P_{i}}{\sigma^{2}}\right) u\left(\widehat{P}_{i}\right) .
$$

In equation (4), $I_{0}$ means the first kind of zero-order improved Bessel function and $u$ stands for the unit step function. The second-order matrix calculated according to equation (3) is

$$
E\left\{\widehat{P}_{i}^{2}\right\}=E\left\{P_{i}^{2}\right\}+2 \sigma^{2} .
$$

Assuming $P_{i}^{2}=0$ and $E\left\{\widehat{P}_{i}^{2}\right\}=2 \sigma^{2}$, the variance estimate $\widehat{\sigma}^{2}$ can be calculated based on

$$
\widehat{\sigma}^{2}=\frac{1}{2 N} \sum_{i=1}^{N} \widehat{P}_{i}^{2}
$$

In the above noise models, the Rician distribution was approximate to the Gaussian distribution when the SNR of the image was high; when the SNR was low, the Rician noise was approximate to the characteristics of DWI noise. In order to eliminate the noise in the DWI-MRI image, the partial differential equation (PDE) model was used for image denoising. Assuming there was a grayscale image, its timeevolution differential equation can be expressed as follows:

$$
\frac{\partial L}{\partial t}=\lambda_{1} L_{\xi \xi}+\lambda_{2} L_{\eta \eta}
$$

In equation (7), $\eta$ indicates the gradient direction of the image; $\xi$ represents the tangent direction of the equal-gray contour of the image; $\lambda_{1}$ and $\lambda_{2}$ stand for the diffusion coefficient in the normal direction and the tangent direction, respectively; and $L_{\xi \xi}$ and $L_{\eta \eta}$ mean the quadratic differential in the normal direction and the tangent direction in the local range, respectively. It could be found that differentiation in the tangent direction was equal to smoothing along the tangent direction, differentiation in the normal direction was equal to blurring the edge of the image, and the diffusion coefficient determined the smoothness of the corresponding direction. In order to control the change of diffusion coefficient through the size of the image gradient mode, a PM model of the anisotropic diffusion model was applied in this study to regularize the image, and its function expression is shown as follows:

$$
\frac{\partial L}{\partial t}=\operatorname{div}[c(|\nabla L|) \nabla L] .
$$

In equation (8), div represents the divergence operator and $\nabla$ stands for the gradient operator. In the calculation of the PM model, the change of $|\nabla L|$ was small in the uniform area of the image, and equation (8) was approximate to the isotropic diffusion; in the boundary area of the image, the change of $|\nabla L|$ was large and $c(|\nabla L|)$ was approximate to 0 , thus preserving the image boundary. However, the gradient change was too large and it was easy to be preserved as the edge of the image if there was a large amount of noise in the image, which affected the noise removal to a certain extent. Therefore, the Catte model was adopted on the basis of the PM model for gradient solution after image filtering, and the function expression of the Catte model is as follows:

$$
\frac{\partial L}{\partial t}=\operatorname{div}\left[c\left(\left|\nabla G_{\sigma} * L\right|^{2}\right) \nabla L\right]
$$

In equation (9), $G_{\sigma}$ represents the Gaussian smoothing kernel. This model could further improve the denoising performance and better retain the edge details of the image.

2.4. Optimization Model Based on CSDCNN Algorithm. The classification method of osteosarcoma pathological images used in this study was designed based on deep learning and data-driven multiclassification methods. The 
CSDCNN algorithm could realize the data-driven method by adopting an advanced enhancement method, and its computing performance was more reliable and efficient. This algorithm could not only automatically adjust the distance of feature space between different categories but also extract high-level semantic features from images, so as to effectively improve the problem of insufficient feature extraction of machine learning algorithms. The CSDCNN algorithm used in this study consisted of an input layer, a convolution layer, and a pooling layer.

During the calculation of the CSDCNN algorithm, the whole MRI image of osteosarcoma patient was first loaded, and then the reduced $224 * 2243$-channel image was output to fit the convolutional layer. The convolution layer applied a configurable convolution kernel to perform convolution operations on the input image. In the convolutional layer, multiple neurons with shared weight parameters formed feature subgraphs, and then a convolutional layer was formed by multiple feature subgraphs together. In this way, links between different levels could be reduced and excessive fitting could be avoided. When the original image reached the convolution layer, the original data were compared with the obtained data convolved by the convolution kernel in terms of the feature mapping. For a neural network, its calculation equation on the two-dimensional tensor can be expressed as follows:

$$
y[a, b]=\sum_{m} \sum_{n} x[a+m, b+n] w[m, n] .
$$

In equation (10), $y[a, b], x[a, b]$, and $w[m, n]$ represent the output two-dimensional tensor, the input two-dimensional tensor, and the weight of the convolution kernel in turn, respectively. For a complete convolutional neural network, each layer contained several feature images. It was assumed that each layer contained $b$ convolution kernels, and each convolution kernel was composed of $m$ groups of parameters. Then, the operation of convolution at each layer can be expressed as follows:

$$
y_{b}^{l}=\sum_{m=1}^{A} w_{b m}^{l} * y_{m}^{l-1}+c_{b}^{l}
$$

In equation (11), $y_{b}^{l}$ stands for the $b$-th feature image of the $l$-th convolution layer; $w_{b m}^{l}$ represents the convolution kernel parameter of the $m$-th feature image of the $l-1$-th convolution layer mapped to the $b$-th feature image of the $l-1$-th convolution layer; $y_{m}^{l-1}$ means the $m$-th feature image of the $l-1$-th convolution layer; and $c_{b}^{l}$ expresses the bias of the $b$-th feature image of the $l$-th convolution layer. The convolution layer of the CSDCNN model used in this study was composed of the ordinary convolution layer and the inception convolution layer. The step size of the ordinary convolution layer was set as 2 , and the convolution kernel sizes of the two convolution layers were $7 \times 7$ and $3 \times 3$, respectively. Convolution operation was a kind of linear transformation. In order to realize multilayer nonlinear features, a nonlinear activation function should be added at the end of each layer of convolution operation. In addition, the sigmoid function was taken as an example, and its expression is

$$
f(x)=\frac{1}{e^{-x}+1} .
$$

The pooling layer belongs to a special convolution, and the training goal of the whole pooling process is to reduce the model parameters and simplify the training model. Besides, pooling operation includes the maximum average pooling and mean pooling. Pooling does not carry out weighting operation, nor does it need activation function, but participates in the design of network mechanism to reduce data dimensions and accelerate calculation speed to prevent overfitting [18]. Maximum pooling can reduce the error caused by the bias of the mean value of the estimate caused by the parameter error of the convolutional layer, and mean pooling can reduce the error caused by the variance of the estimate caused by the neighborhood size limitation. The two pooling methods are designed to better retain the image texture and image background information (Figure 1).

The last step of the CSDCNN algorithm was to set a highprecision loss multiple classifier and constraint equation. Assuming there were $N$ input images, the mark of each image can be expressed as

$$
Y_{j} \in\{0,1,2, \ldots, k, \ldots, K\}, \quad K>2 .
$$

For a given test image $X_{j}$, a hypothesis function was applied to estimate the probability value $p$ of each category $g$, so the expression of the function $H_{\theta}\left(X_{j}\right)$ is supposed as follows:

$$
\begin{aligned}
H_{\theta}\left(X_{j}\right) & =\left[\begin{array}{c}
p\left(Y_{j}=0 \mid X_{j} ; \theta\right) \\
p\left(Y_{j}=1 \mid X_{j} ; \theta\right) \\
\vdots \\
p\left(Y_{j}=K \mid X_{j} ; \theta\right)
\end{array}\right] \\
& =\frac{1}{\sum_{K=0}^{K} e^{\theta_{g}^{T} X_{j}}}\left[\begin{array}{c}
e^{\theta_{0}^{T} X_{j}} \\
e^{\theta_{1}^{T} X_{j}} \\
\vdots \\
e^{\theta_{k}^{T} X_{j}}
\end{array}\right] .
\end{aligned}
$$

In equation (14), $1 / \sum_{K=0}^{K} e^{\theta_{g}^{T} X_{j}}$ stands for the normalization of probability distribution, and the loss function can be expressed as

$$
R(X, Y, \theta)=-\frac{1}{E}\left[\sum_{j=1}^{E} \sum_{K=0}^{K} 1\left\{Y_{j}=r\right\} \log \frac{e^{\theta_{k}^{T} X_{j}}}{1 / \sum_{K=0}^{K} e^{\theta_{k}^{T} X_{j}}}\right] .
$$

In the above equation, $1\left\{Y_{j}=r\right\}$ represents the indicative function. In fine-grained images with a small gap between classes, the data feature space would be compressed 


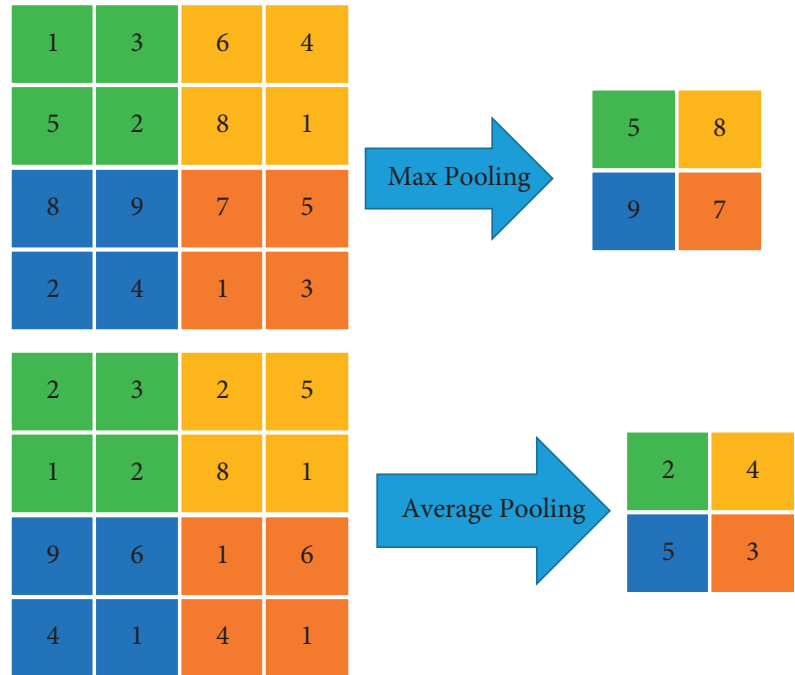

FIGURE 1: Schematic diagram of maximum and mean pooling. the difference between classes, a loss layer considering the prior category should be added to the loss layer after the classifier.

It was assumed that $X_{j}, P_{j}^{+}, P_{j}^{-}$, and $v_{j}$ represent the original image, the same small class of image, the same big class of image, and different big class of image, respectively. During calculation, the Euclidean distance of the feature space of the same image should be reduced, and the Euclidean distance of the feature space of the image of different classes should be increased, namely,

$$
Z\left(X_{j}, P_{j}^{+}\right)+w_{1}<Z\left(X_{j}, P_{j}^{-}\right)+w_{2}<Z\left(X_{j}, v_{j}\right) .
$$

In equation (16), $Z$ means the Euclidean distance between the features of two images; $w_{1}$ and $w_{2}$ are super parameters, which were employed to control the distance interval between images. The Hinge function was used as its error function:

to a very small corner, and the feature difference between classes could not be effectively preserved. In order to retain

$$
\begin{aligned}
U_{t}\left(X_{j}, P_{j}^{+}, P_{j}^{-}, v_{j}, w_{1}, w_{2}\right)= & \frac{1}{2 E} \sum_{j=1}^{E} \max \left\{0, Z\left(X_{j}, P_{j}^{+}\right)-Z\left(X_{j}, P_{j}^{-}\right)+w_{1}-w_{2}\right\} \\
& +\frac{1}{2 E} \sum_{j=1}^{E} \max \left\{0, Z\left(X_{j}, P_{j}^{-}\right)-Z\left(X_{j}, v_{j}\right)+w_{2}\right\} .
\end{aligned}
$$

Combined with equation (14), the new loss layer could be obtained:

$$
U=\lambda R(X, Y, \theta)+(1-\lambda) U_{t}\left(X_{j}, P_{j}^{+}, P_{j}^{-}, v_{j}, w_{1}, w_{2}\right) .
$$

The workflow of the CSDCNN algorithm consisted of training stage, validation stage, and testing stage (Figure 2), and the main purpose of the training stage was to learn enough feature representation and to optimize the distance of the feature space of different categories. The purpose of the training stage was to fine-tune the parameters and to select the characteristics of each epoch, so as to adjust the search for the best multiclassification model for osteosarcoma and corresponding histopathological images. The purpose of the test stage was to evaluate the computational performance of the CSDCNN algorithm. In this study, the learning rate of CSDCNN was set as 0.01 and the number of iterations was set as 52,000 .

2.5. Image Quality Evaluation. For all patients with osteosarcoma in this study, two methods of subjective evaluation and objective evaluation were used for the processing effect of DWI-MRI images. Subjective evaluation was mainly observed by naked eyes, which was easily affected by the subjective consciousness of the evaluator. Therefore, it lacked a quantitative standard to measure the quality of the image. On this basis, some objective evaluation indicators were introduced for evaluation, including peak SNR (PSNR), mean square error (MSE), and edge preserve index (EPI), and the three indicator functions are displayed as follows:

$$
\begin{aligned}
\text { PSNR } & =10 \lg \left[\frac{p e_{\max }^{2}}{(1 / h \cdot l) \sum_{\varsigma=1, \tau=1}^{h, l}[f(\varsigma, \tau)-\widehat{f}(\varsigma, \tau)]^{2}}\right] \\
\mathrm{MSE} & =\frac{1}{h \cdot l} \sum_{\varsigma=1, \tau=1}^{h, l}[f(\varsigma, \tau)-\widehat{f}(\varsigma, \tau)]^{2},
\end{aligned}
$$




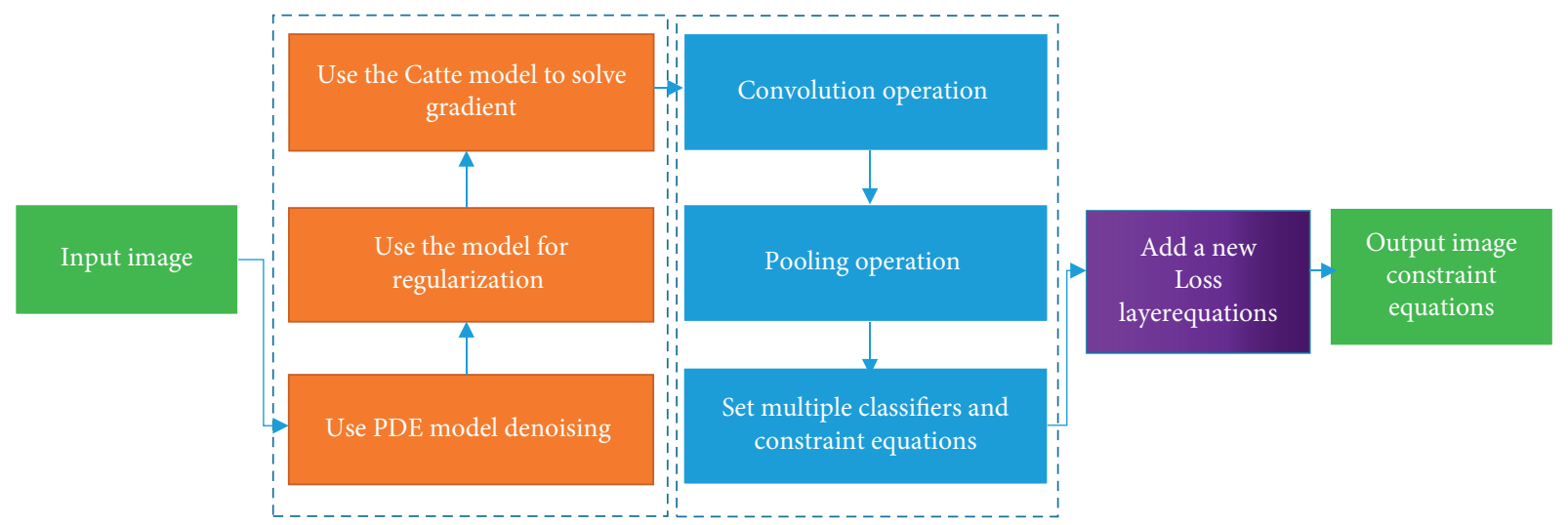

Figure 2: Calculation flow of CSDCNN algorithm.

$$
\mathrm{EPI}=\frac{\sum_{\varsigma=1, \tau=1}[|\widehat{f}(\varsigma+1, \tau)-\widehat{f}(\varsigma, \tau)|+|\widehat{f}(\varsigma, \tau+1)-\hat{f}(\varsigma, \tau)|]}{\sum_{\varsigma=1, \tau=1}[|f(\varsigma+1, \tau)-f(\varsigma, \tau)|+|f(\varsigma, \tau+1)-f(\varsigma, \tau)|]}
$$

In equations (19)-(21), $h$ and $l$ represent the height and width of the image in turn, respectively, $f$ indicates the goldstandard image, $\widehat{f}$ means the denoised image, and $p e_{\max }$ stands for the maximum peak value, which is 1 in this study.

2.6. Evaluation of Diagnostic Accuracy. In order to diagnose the performance of the CSDCNN algorithm in medical images of patients with osteosarcoma, the accuracy, recall rate, precision rate, and $F_{1}$ score were adopted in this study to evaluate the evaluation effect of the algorithm, and SegNet, LeNet, and AlexNet algorithms were introduced for comparison. The calculation equations of the four evaluation indicators are shown as follows:

$$
\begin{aligned}
\text { accuracy } & =\frac{\mathrm{TP}+\mathrm{TN}}{\mathrm{TP}+\mathrm{TN}+\mathrm{FP}+\mathrm{FN}^{\prime}}, \\
\text { recall } & =\frac{\mathrm{TP}}{\mathrm{TP}+\mathrm{FN}}, \\
\text { precise } & =\frac{\mathrm{TP}}{\mathrm{FP}+\mathrm{TP}}, \\
F_{1} & =\frac{2(\text { recall } \times \text { precise })}{\text { recall }+ \text { precise }} .
\end{aligned}
$$

In equations (22)-(25), Accuracy, Recall, and Precise stand for accuracy, recall rate, and precision in sequence. Furthermore, TP means the number of correctly classified positive samples, TN represents the number of correctly classified negative samples, FP indicates the number of misclassified negative samples, and FN expresses the number of misclassified positive samples. Furthermore, $F_{1}$ score is a weighted mean of model precision and recall rate, with the value range of $0-1$.

2.7. ADC Value Measurement. The maximum tumor layer of all patients with osteosarcoma was selected to measure the $A D C$ value before and after neoadjuvant chemotherapy. The mean of 3 measurements was taken as $\mathrm{ADC}_{\text {mean }}$ and the minimum value was taken as $\mathrm{ADC}_{\text {min }}$. The region of interest in the patient images was drawn by referring to T2WI and enhanced images, and then the region of interest was placed in the solid region of the tumor. What's more, it should avoid necrosis, bleeding, cystic degeneration, and neoplastic bone components. Then, the ADC change rate was automatically generated, and its calculation equation is as follows:

$$
\mathrm{ADC}=\frac{\mathrm{ADC}_{\mathrm{after}}-\mathrm{ADC}_{\text {before }}}{\mathrm{ADC}_{\text {before }}} \times 100 \% .
$$

In equation (26), $\mathrm{ADC}_{\text {before }}$ and $\mathrm{ADC}_{\text {after }}$ stand for the ADC value of patients with osteosarcoma before neoadjuvant therapy and the ADC value of patients with osteosarcoma after neoadjuvant therapy, respectively.

2.8. Statistical Analysis. SPSS19.0 software was used for data processing in this study. Measurement data were represented by $\bar{x}$, which were detected by $t$-tests of two independent samples. Besides, count data were tested by $\chi^{2}$, and the correlation of parameters was analyzed by Pearson. In addition, $P<0.05$ indicated that the difference was statistically substantial.

\section{Experimental Results}

3.1. General Clinical Data of the Patient. Among the 48 patients participating in the study, 30 were males, with an average age of $16 \pm 2.1$ years, and 10 were females, with an average age of $17 \pm 1.5$ years. All 48 patients involved in this study underwent puncture biopsy after DWI-MRI examinations and were analyzed. Pathological sections and immunohistochemical analysis results disclosed that 41 patients had osteosarcoma, among which 16 cases had lesions at the lower end of the femur, 10 at the upper end of the humerus, 7 at the upper end of the tibia, and 8 at the upper end of the fibula (Figure 3). 


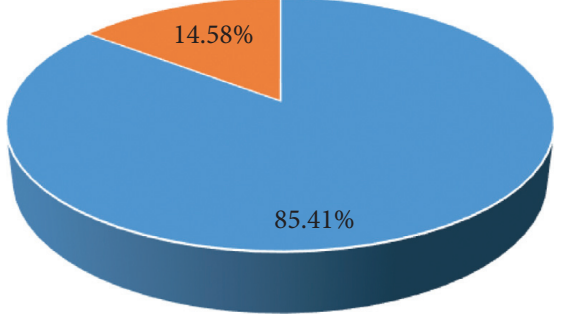

Osteosarcoma
Non-osteosarcoma

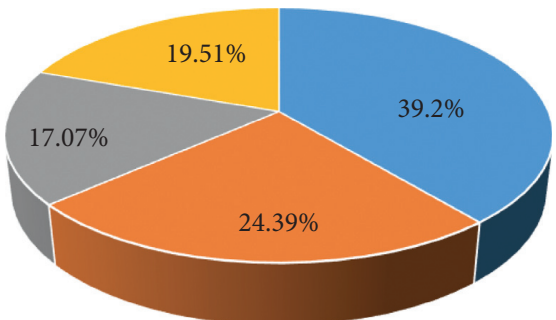

Lower end of femur

Upper humerus

Upper Tibia

(a)

(b)

FIGURE 3: General information of the patient: (a) the prevalence of osteosarcoma and (b) the lesion site of osteosarcoma.

3.2. DWI-MRI Image Denoising Results of Patients with Osteosarcoma. DWI-MRI images of patients with osteosarcoma used in this study were all clinical scanning data, and the scanning parameters were as follows: $\mathrm{TR}=3,800 \mathrm{~ms}$, $\mathrm{TE}=88 \mathrm{~ms}, b=800 \mathrm{~s} / \mathrm{mm}^{2}$, pixel size was $0.9 \times 0.9 \times 2.5 \mathrm{~mm}^{3}$, and acquisition time was 6.3 minutes, with a total of 48 scanning layers. Besides, DWI images of patients were collected from 35 gradient directions. After the scanning, the DWI images of the patients were denoised, and the results are shown in Figure 4. The original image was locally enlarged and compared with the image regularized by the PM algorithm and solved by gradient solution after image filtering with the Catte model (Figure 5). It was found that there was obvious noise in the original DWI image of the patient, but the noise was well filtered out after the denoising algorithm and the image information was retained intact.

\subsection{Discrimination of Lesion Sites in Patients with Osteo-} sarcoma Based on Different Algorithms. Different algorithms were used to distinguish the lesion location on the DWIMRI image of patients with sarcoma, as shown in Figure 6 (male patients aged 18 years). T2WI showed low signal, periosteal reaction showed cortical thickening, tumor margins had slightly enhanced tumor, and bone strengthening was not obvious. Data were taken to enhance the generalization CSDCNN model, including random distortion, random filtering, random brightness, and contrast. From the DWI-MRI images obtained after discrimination by different algorithms, the CSDCNN algorithm used in this study could be applied to accurately diagnose the location of the lesions in patients with osteosarcoma, and even the boundary between the lesions and normal tissues had a high degree of overlap. The CSDCNN algorithm also achieved the precise positioning of the lesions, indicating that the CSDCNN algorithm had great advantages in the identification of lesions in patients with osteosarcoma.

3.4. Evaluation Results of Image Quality. Since the processing effect of different algorithms based on visual discrimination was easily affected by the subjective consciousness of the evaluator, the PSNR, MSE, and EPI were adopted to objectively evaluate the processing effect of different algorithms. The PSNR represented the ratio between useful signal power and noise power in an image, which was inversely proportional to the degree of image distortion. The larger the PSNR, the smaller the image distortion. The PSNR values after image processing by different algorithms are displayed in Figure 7(a). The results suggested that the PSNR value of the images processed by the CSDCNN algorithm was higher obviously than the value of SegNet, LeNet, and AlexNet algorithms $(P<0.05)$ after the DWI-MRI images of patients were processed by different algorithms, suggesting that the image distortion processed by the CSDCNN algorithm was smaller.

The MSE was the degree of gray change between the processed image and the ideal image. The smaller the MSE, the closer the processed image to the real image. MSE values after image processing by different algorithms are shown in Figure 7(b), indicating that the MSE value of the images processed by the CSDCNN algorithm rose hugely in contrast to the value of other algorithms after the processing of patients' DWI-MRI images by different algorithms $(P<0.05)$. Thus, it revealed that the accuracy of the images processed by the CSDCNN algorithm was higher.

The EPI reflected the degree of image edge protection, and its value range was $0-1$. The larger the value, the better the edge protection. Figure 7 (c) shows the EPI values after image processing by different algorithms. It was found that after the DWI-MRI images of patients was processed by different algorithms, the EPI value of the images processed by the CSDCNN algorithm elevated greatly compared with other algorithms $(P<0.05)$, meaning that the CSDCNN algorithm had better protection of image details after processing.

\subsection{Evaluation of Diagnostic Accuracy Based on Different} Algorithms. Different algorithms were adopted to evaluate the detection rate of osteosarcoma, a total of 40 cases with osteosarcoma were detected by the CSDCNN algorithm, and 1 case was missed. Compared with the other three algorithms, the diagnosis results were hugely different $(P<0.05)$. Accuracy, Recall, Precise, and $F_{1}$ score were adopted to 


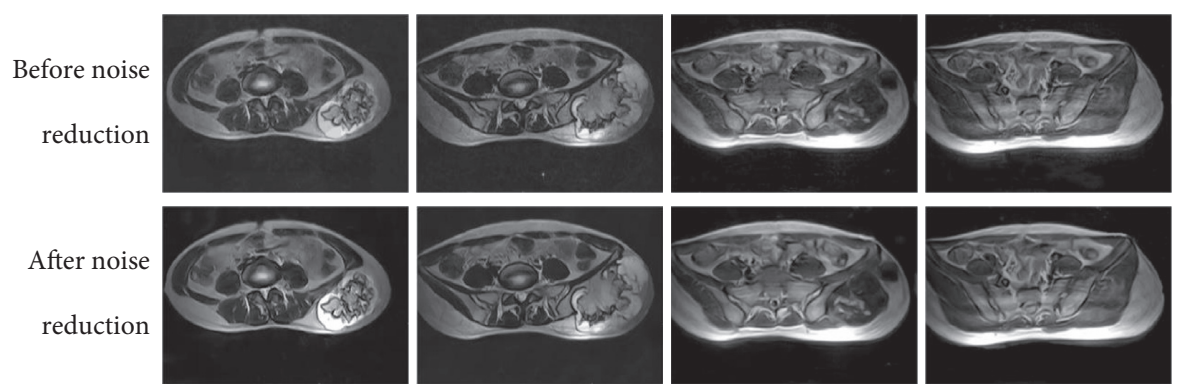

Figure 4: DWI-MRI image denoising effect of patients with osteosarcoma.

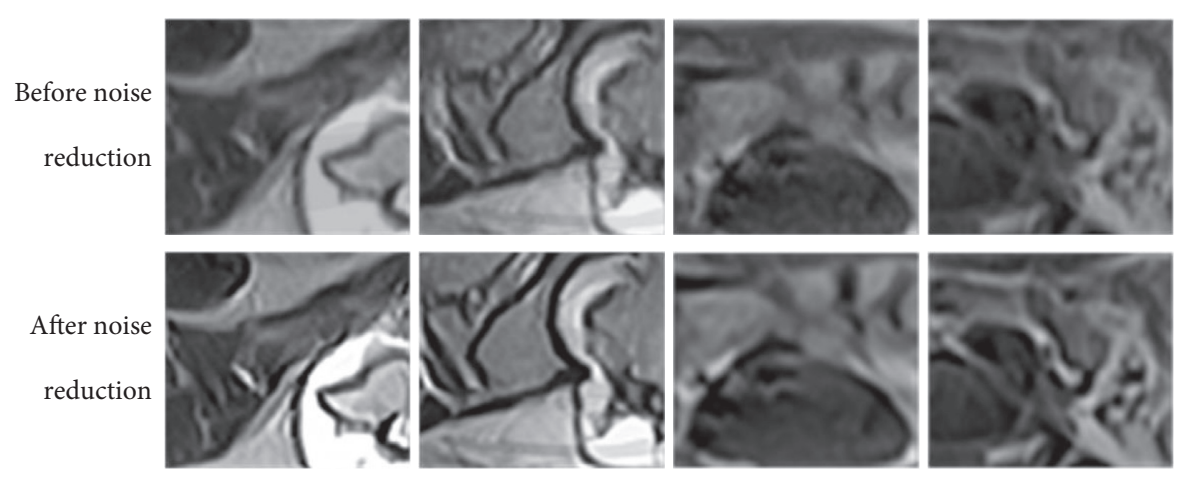

FIGURE 5: Local magnification effect before and after denoising of DWI-MRI images of patients with osteosarcoma.

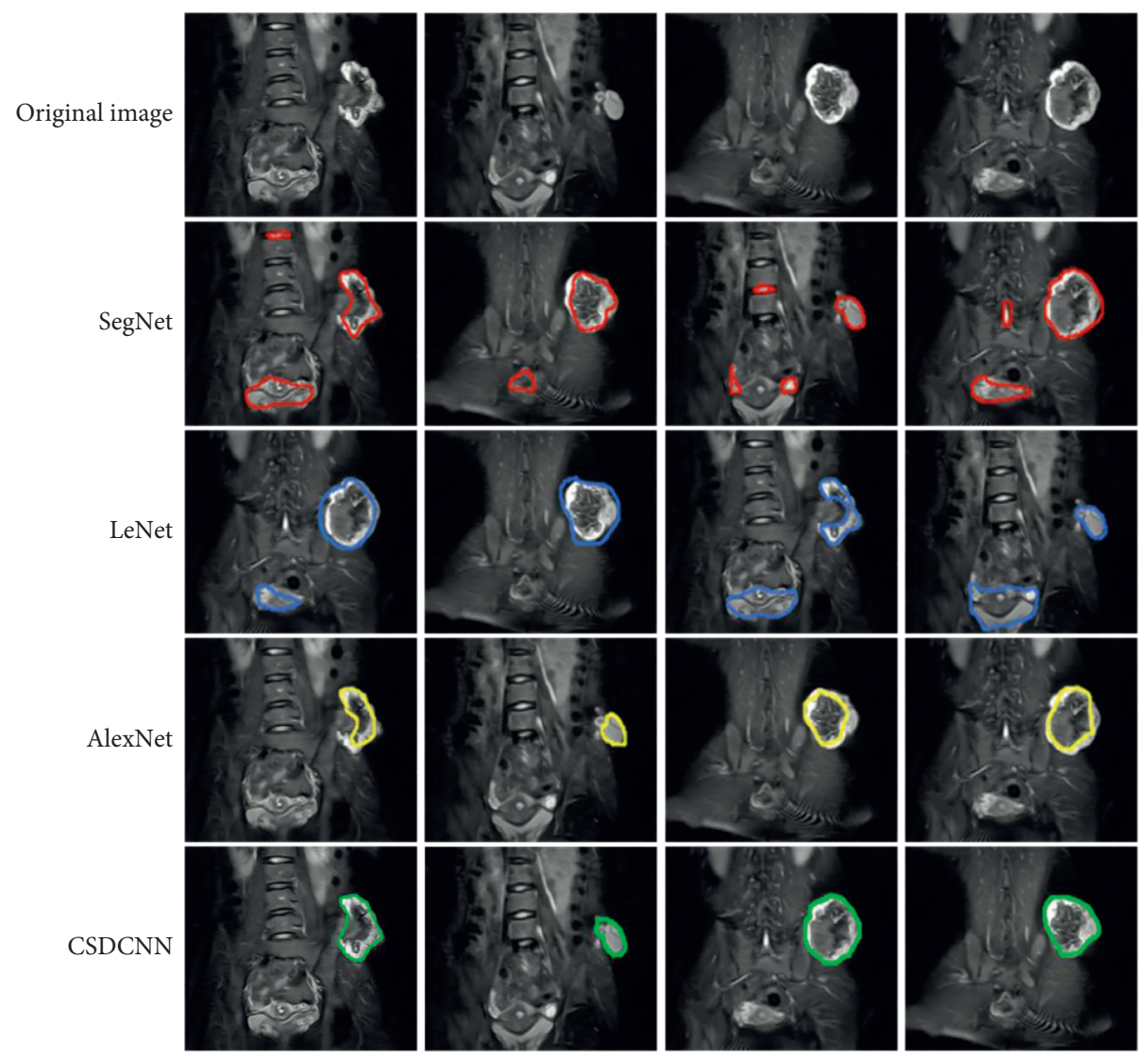

FIGURE 6: Discriminant results of lesion sites in patients with osteosarcoma based on different algorithms. 


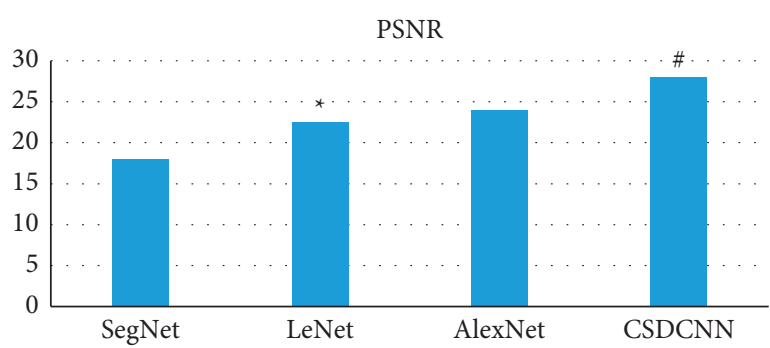

(a)

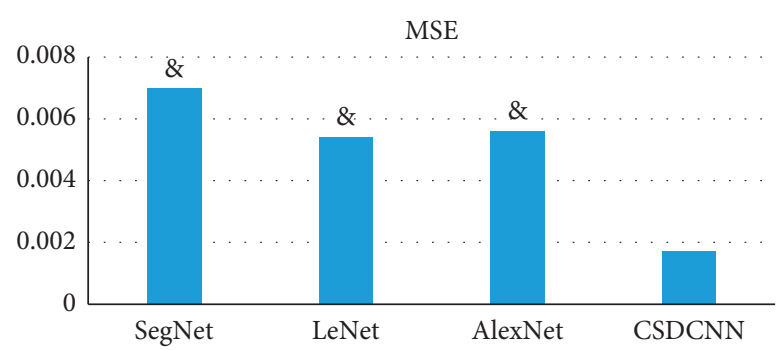

(b)

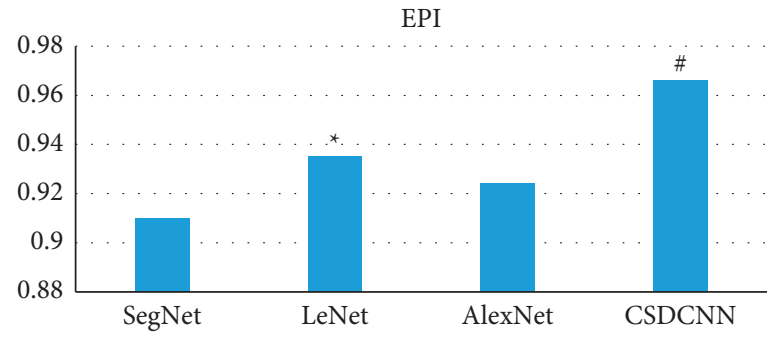

(c)

Figure 7: Evaluation of DWI-MRI image processing effect of different algorithms in patients with osteosarcoma. (Note. Compared with SegNet, ${ }^{*} P<0.05$; compared with AlexNet, ${ }^{\#} P<0.05$; compared with CSDCNN, ${ }^{*} P<0.05$.)

evaluate the diagnostic effects of different algorithms on osteosarcoma; the results showed that the Accuracy of the CSDCNN algorithm was 0.9812, Recall was 0.9011, Precise was 0.8563 , and $F_{1}$ score was 0.9316 . The above four indicators were significantly better than other algorithms, and the difference was statistically significant $(P<0.05)$, suggesting that the CSDCNN algorithm had a better diagnostic effect on patients with osteosarcoma (Figure 8).

\subsection{Effect Evaluation of Neoadjuvant Chemotherapy Based on} Different Algorithms. In this study, the ADC value was adopted to evaluate the therapeutic effect of neoadjuvant chemotherapy. The change of ADC value could be used to judge the degree of water molecular diffusion, which indirectly reflected the therapeutic effect of osteosarcoma. The 41 patients were rolled randomly into 4 groups, including 10 cases in the SegNet algorithm adjuvant chemotherapy group, 10 cases in the LeNet algorithm adjuvant chemotherapy group, 10 cases in the AlexNet algorithm adjuvant chemotherapy group, and 11 cases in the CSDCNN adjuvant chemotherapy group. The results indicated that there was no marked difference in ADC values of the 4 groups before chemotherapy $(P>0.05)$. After they were treated with neoadjuvant chemotherapy, the $\mathrm{ADC}_{\text {mean }}$ value of the SegNet adjuvant chemotherapy group was $1.32 \pm 0.19$, and the $\mathrm{ADC}_{\text {min }}$ value was $0.95 \pm 0.14$; the $\mathrm{ADC}_{\text {mean }}$ value of the LeNet adjuvant chemotherapy group was $1.35 \pm 0.22$, and the $\mathrm{ADC}_{\min }$ value was $0.98 \pm 0.19$; the $\mathrm{ADC}_{\text {mean }}$ value of the AlexNet adjuvant chemotherapy group was $1.41 \pm 0.21$, and the $\mathrm{ADC}_{\text {min }}$ value was $0.96 \pm 0.21$; the $\mathrm{ADC}_{\text {mean }}$ value of the CSDCNN adjuvant chemotherapy group was $1.66 \pm 0.17$, and the $\mathrm{ADC}_{\min }$ value was $1.33 \pm 0.15$. The two indexes were significantly higher than the other three groups, and the difference was statistically significant $(P<0.05)$. It was suggested that the CSDCNN algorithm was better for DWIMRI images of patients with osteosarcoma after preanalysis and discrimination (Figure 9).

\section{Discussion}

Osteosarcoma is composed of highly heteromorphic osteoblastic sarcoma cells and the osteoid tissue and tumor bone tissue formed by them. It usually occurs in adolescents and children, which is prone to distant metastasis due to its high degree of malignancy [19]. The current clinical treatment of osteosarcoma is a combination of chemotherapy and surgery. Since the comprehensive treatment of "neoadjuvant chemotherapy + extensive resection + postoperative chemotherapy" has been extensively used, the limb salvage rate and five-year survival rate of patients have been greatly improved [20]. Research investigations have pointed out that the incidence of osteosarcoma is increasing year by year. Therefore, early diagnosis and treatment largely determines the prognosis [21], and the treatment strategy for the clinic can be adjusted based on the accurate assessment of the focus of the disease in the early stage of the disease. It usually leads to substantial differences in the results because of the different methods of obtaining materials from different patients' pathological sites. Therefore, imaging examination is very important for the early diagnosis of osteosarcoma and the evaluation of the therapeutic effect. Due to its advantages of multidirectional parameter imaging and high soft tissue resolution, MRI can not only accurately reflect the characteristics of the patient's lesion but also reflect the hemodynamic changes of the lesion through dynamic enhancement [22]. With the continuous development of MRI technology, the application of DWI technology has played a vital role in the detection of osteosarcoma lesions and the determination of osteosarcoma boundaries. 


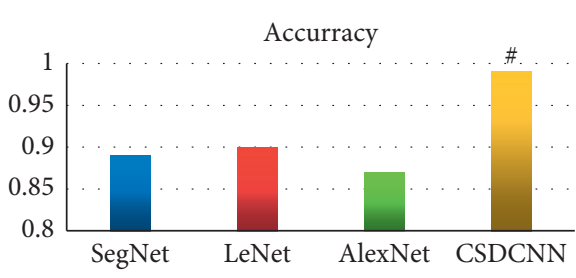

(a)

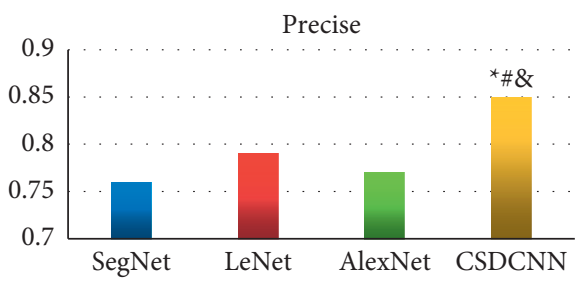

(c)

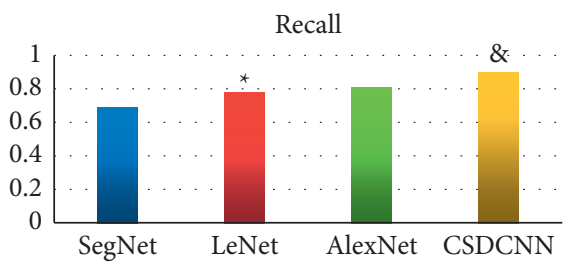

(b)

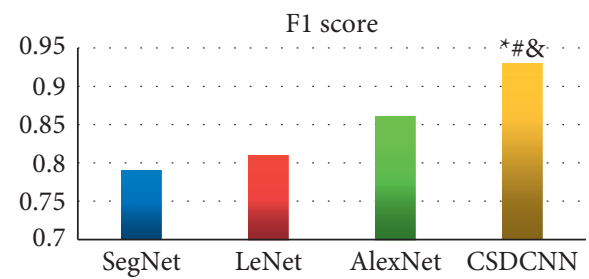

(d)

Figure 8: Evaluation of diagnostic effect of different algorithms on patients with osteosarcoma. (Note. Compared with SegNet, ${ }^{*} P<0.05$; compared with LeNet, ${ }^{\#} P<0.05$; compared with AlexNet, ${ }^{8} P<0.05$.)

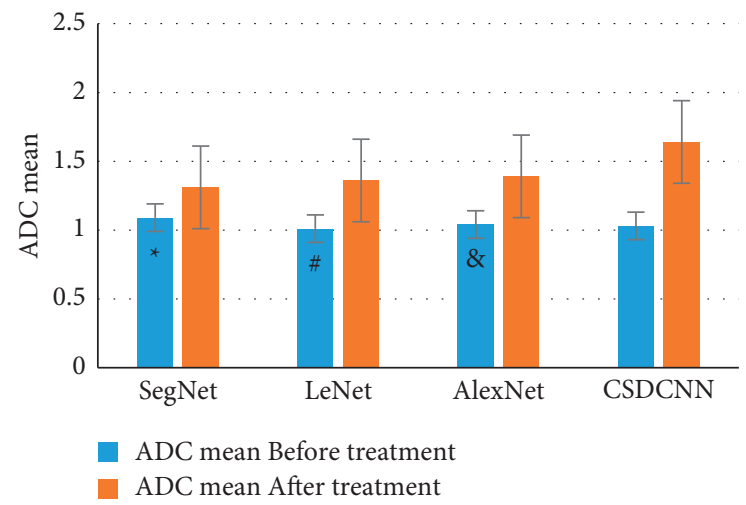

(a)

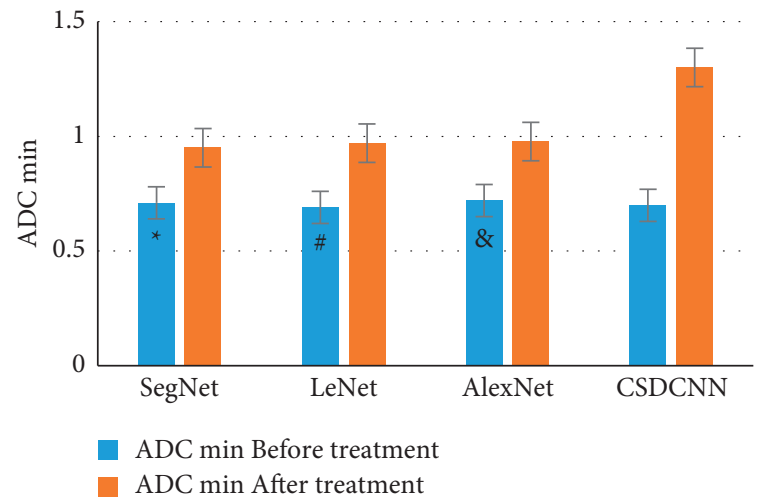

(b)

FIGURE 9: Comparison on ADC values before and after neoadjuvant chemotherapy for osteosarcoma based on different algorithms. ${ }^{*}$ indicates that there is a statistical difference in the ADC value between the CSDCNN adjuvant chemotherapy group and the SegNet adjuvant chemotherapy group after treatment; "indicates that there is a statistical difference in the ADC value between the CSDCNN adjuvant chemotherapy group and the LeNet adjuvant chemotherapy group after treatment; ${ }^{\&}$ indicates that there is a statistical difference in the ADC value between the CSDCNN adjuvant chemotherapy group and the AlexNet adjuvant chemotherapy group after treatment.)

However, the SNR is low and the image quality is poor due to the large susceptibility distortion artifacts of routine DWI imaging [23], which makes it difficult for clinicians to make accurate diagnosis based on patient images. With the continuous development of computer technology, many machine learning and deep learning algorithms have been applied to the field of image recognition. The purpose is to reduce the repeated work of clinicians, relieve the pressure of reading pictures, help doctors make more reliable and objective diagnosis results, and assist in the completion of clinical treatment of various diseases [24]. Therefore, the use of computer technology to mine and analyze clinical data is of great significance to the development of clinical medicine.

In order to improve the efficiency of the early imaging diagnosis of patients with osteosarcoma and the effect of neoadjuvant chemotherapy based on the results of imaging examinations, the patient DWI-MRI image noise model was first established, the PDE was adopted to denoise the image, and the image was filtered by the Catte model for gradient solution. The DWI-MRI image discrimination model of patients with osteosarcoma based on the CSDCNN algorithm was established after the regularization process. 48 patients with suspected osteosarcoma were selected as the research objects in this study. Through the processing of DWI-MRI images of the patients, it was found that the CSDCNN algorithm realized the precise location of the lesions in patients with osteosarcoma, and the PSNR, MSE, and EPI values after image processing were significantly higher than those of the SegNet, LeNet, and AlexNet algorithms $(P<0.05)$. Thus, it showed that the CSDCNN algorithm had a better discrimination effect on DWI-MRI images of patients with osteosarcoma. By evaluating the accuracy of the diagnosis of osteosarcoma patients with different algorithms, the CSDCNN algorithm had only 
one missed diagnosis, and the diagnosis result was similar to the pathological examination result. This suggested that the CSDCNN algorithm was more effective in the diagnosis of patients with osteosarcoma than the SegNet, LeNet, and AlexNet algorithms, and this was similar to the results of $\mathrm{Fu}$ et al. [25]. Finally, neoadjuvant chemotherapy was performed on the imaging results processed by different algorithms, finding that the $\mathrm{ADC}_{\text {mean }}$ and $\mathrm{ADC}_{\text {min }}$ values of the neoadjuvant chemotherapy group based on the CSDCNN algorithm were greater remarkably than those of the other three groups. Therefore, it was suggested that the CSDSNN algorithm could be used for prediscriminant analysis of DWI-MRI images of patients, which was helpful to improve the therapeutic effect of neoadjuvant chemotherapy.

\section{Conclusion}

In this study, the DWI-MRI images of patients with osteosarcoma were first processed to regularize, in order to eliminate the noise in the images. Then, the DWI-MRI image discrimination model of the CSDCNN algorithm was established and applied to the diagnosis and treatment of patients with osteosarcoma. The results of this study found that the CSDCNN algorithm had substantial results in discriminating DWI-MRI images of patients with osteosarcoma, which could significantly improve the accuracy of the diagnosis of osteosarcoma and assist clinicians in the imaging diagnosis of patients with osteosarcoma. Moreover, the diagnostic results based on this algorithm could obtain better therapeutic effect of neoadjuvant chemotherapy. However, there are still some shortcomings in this study. The sample size of this study is small, and the effect of extensive resection and postoperative chemotherapy has not been evaluated, which needs to be confirmed by more largesample clinical studies in future studies.

\section{Data Availability}

The data used to support the findings of this study are available from the corresponding author upon request.

\section{Conflicts of Interest}

The authors declare no conflicts of interest.

\section{References}

[1] L. Kager, G. Tamamyan, and S. Bielack, "Novel insights and therapeutic interventions for pediatric osteosarcoma," Future Oncology, vol. 13, no. 4, pp. 357-368, 2017.

[2] F. Cersosimo, S. Lonardi, G. Bernardini et al., "Tumor-associated macrophages in osteosarcoma: from mechanisms to therapy," International Journal of Molecular Sciences, vol. 21, no. 15 , p. $5207,2020$.

[3] V. Ramanujan, A. Krishnamurthy, K. Venkataramani, and C. Kumar, "Pulmonary metastasectomy in primary extremity osteosarcoma: choosing wisely, along with a brief review of literature," Indian Journal of Cancer, vol. 57, no. 2, pp. 172-181, 2020.
[4] K. Mardanpour, M. Rahbar, S. Mardanpour, S. Khazaei, and M. Rezaei, "Co-expression of Epstein-Barr virus-encoded RNA1 and viral latent membrane protein 1 in osteosarcoma: a novel insight of predictive markers," Tumour biology: The Journal of the International Society for Oncodevelopmental Biology and Medicine, vol. 42, no. 11, Article ID 1010428320974247, 2020.

[5] C. Tempelaere, D. Biau, A. Babinet, and P. Anract, "Osteosarcoma after the age of fifty: a clinicopathological study," European Journal of Surgical Oncology, vol. 45, no. 7, pp. 1288-1292, 2019.

[6] C. Hongsaprabhas, W. Chenboonthai, P. Suvaraksakul, and C. Charoenlap, "Rotationplasty for unplanned fixation of pathological fracture distal femoral osteosarcoma," Case Report in Orthopedics, vol. 2020, Article ID 8813619, 5 pages, 2020.

[7] J. Zhu, T. He, Z. Wei, and Y. Wang, "Retrospective analysis of the effect of treatment of osteosarcoma complicated by pathological fracture by neoadjuvant chemotherapy combined with limb salvage surgery," Journal of B.U.ON.: Official Journal of the Balkan Union of Oncology, vol. 23, no. 6, pp. 1809-1815, 2018.

[8] Y. Wang, W. Guo, D. Shen et al., "Surgical treatment of primary osteosarcoma of the sacrum," Spine, vol. 42 , no. 16, pp. 1207-1213, 2017.

[9] P. Lin, P. F. Yang, S. Chen et al., "A Delta-radiomics model for preoperative evaluation of Neoadjuvant chemotherapy response in high-grade osteosarcoma," Cancer Imaging, vol. 20, no. 1,2020 .

[10] A. Takeuchi, N. Yamamoto, K. Hayashi et al., "Joint-preservation surgery for pediatric osteosarcoma of the knee joint," Cancer and Metastasis Reviews, vol. 38, no. 4, pp. 709-722, 2019.

[11] Y. Tsuda, K. Tsoi, M. C. Parry et al., "Impact of chemotherapyinduced necrosis on event-free and overall survival after preoperative MAP chemotherapy in patients with primary high-grade localized osteosarcoma," The Bone \& Joint Journal, vol. 102-B, no. 6, pp. 795-803, 2020.

[12] J. M. Cates and W. D. Dupont, "Cytologic anaplasia is a prognostic factor in osteosarcoma biopsies, but mitotic rate or extent of spontaneous tumor necrosis are not: a critique of the College of American Pathologists Bone Biopsy template," Modern Pathology, vol. 30, no. 1, pp. 52-59, 2017.

[13] Z. Luo, W. Chen, X. Shen et al., "CT and MRI features of calvarium and skull base osteosarcoma (CSBO)," British Journal of Radiology, vol. 93, no. 1105, Article ID 20190653, 2020.

[14] M. M. Saleh, T. M. Abdelrahman, Y. Madney, G. Mohamed, A. M. Shokry, and A. F. Moustafa, "Multiparametric MRI with diffusion-weighted imaging in predicting response to chemotherapy in cases of osteosarcoma and Ewing's sarcoma," British Journal of Radiology, vol. 93, no. 1115, Article ID 20200257, 2020.

[15] A. J. Degnan, C. Y. Chung, and A. J. Shah, "Quantitative diffusion-weighted magnetic resonance imaging assessment of chemotherapy treatment response of pediatric osteosarcoma and Ewing sarcoma malignant bone tumors," Clinical Imaging, vol. 47, pp. 9-13, 2018.

[16] S. K. Lee, W. H. Jee, C. K. Jung, S. A. Im, N. G. Chung, and Y. G. Chung, "Prediction of poor responders to neoadjuvant chemotherapy in patients with osteosarcoma: additive value of diffusion-weighted MRI including volumetric analysis to standard MRI at 3T," PLoS One, vol. 15, no. 3, Article ID e0229983., 2020. 
[17] W. Yuan, Q. Yu, Z. Wang, J. Huang, J. Wang, and L. Long, "Efficacy of diffusion-weighted imaging in neoadjuvant chemotherapy for osteosarcoma: a systematic review and meta-analysis," Academic Radiology, vol. 18, no. 20, pp. S1076-S6332, 2020.

[18] C. Guo, J. Lu, Z. Tian, W. Guo, and A. Darvishan, "Optimization of critical parameters of PEM fuel cell using TLBODE based on Elman neural network," Energy Conversion and Management, vol. 183, no. MAR, pp. 149-158, 2019.

[19] F. Pu, F. Chen, Z. Zhang, J. Liu, and Z. Shao, "Information transfer and biological significance of neoplastic exosomes in the tumor microenvironment of osteosarcoma," OncoTargets and Therapy, vol. 13, pp. 8931-8940, 2020.

[20] S. A. Traven, D. L. Brinton, Z. J. Walton, and L. R. Leddy, "A propensity-score matched analysis of limb salvage vs amputation for osteosarcoma," Journal of Surgical Oncology, vol. 120, no. 7, pp. 1252-1258, 2019.

[21] I. Corre, F. Verrecchia, V. Crenn, F. Redini, and V. Trichet, "The osteosarcoma microenvironment: a complex but targetable ecosystem," Cells, vol. 9, no. 4, 2020.

[22] M. Sue, T. Oda, Y. Sasaki, A. Kameta, Y. Okada, and I. Ogura, "Osteosarcoma of the mandible: a case report with CT, MRI and scintigraphy," Chinese Journal of Dental Research, vol. 20, no. 3, pp. 169-172, 2017.

[23] E. K. Baidya, D. Kandasamy, R. Yadav, S. Bakhshi, R. Sharma, and A. Mehndiratta, "Automatic segmentation and RECIST score evaluation in osteosarcoma using diffusion MRI: a computer aided system process," European Journal of Radiology, vol. 133, Article ID 109359, 2020.

[24] S. Guo, R. Chen, H. Li, T. Zhang, and Y. Liu, "Identify severity bug report with distribution imbalance by CR-SMOTE and ELM," International Journal of Software Engineering and Knowledge Engineering, vol. 29, no. 2, pp. 139-175, 2019.

[25] Y. Fu, P. Xue, H. Ji, W. Cui, and E. Dong, "Deep model with Siamese network for viable and necrotic tumor regions assessment in osteosarcoma," Medical Physics, vol. 47, no. 10, pp. 4895-4905, 2020. 